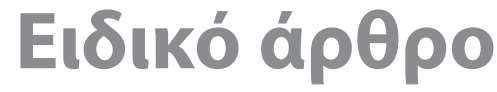 Special article
}

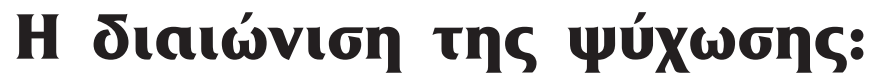

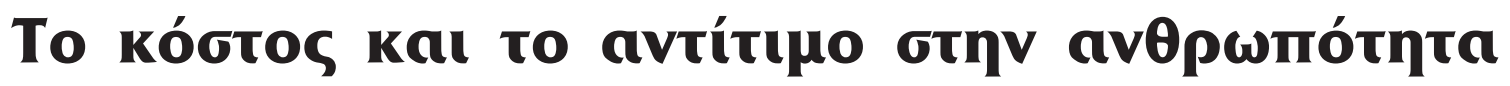

\author{
O. Гıமтákoc

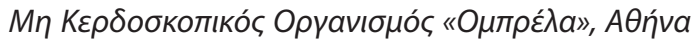 \\ Uuxıатрıкń 2018, 29:316-326
}

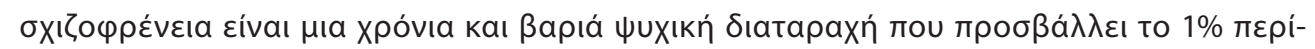

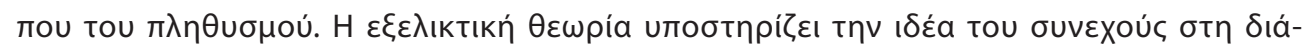

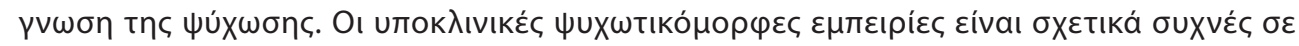

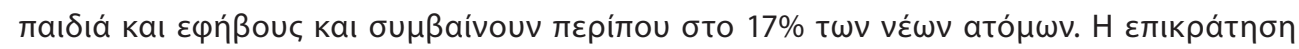

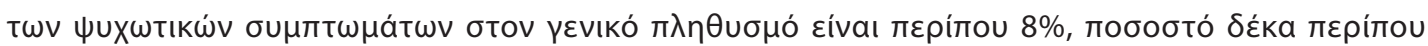

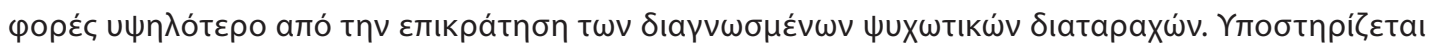

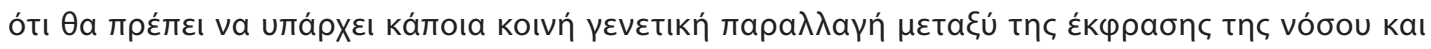

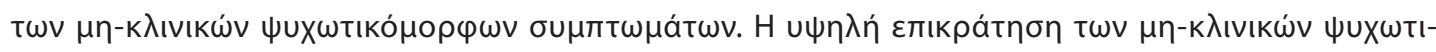

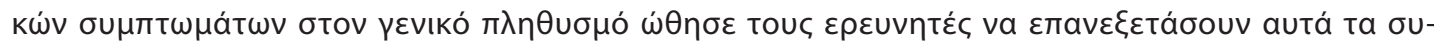

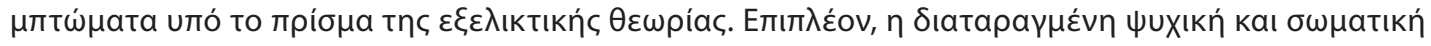

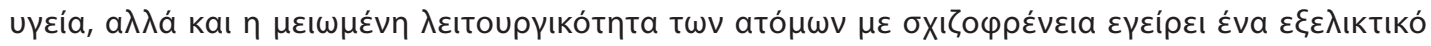

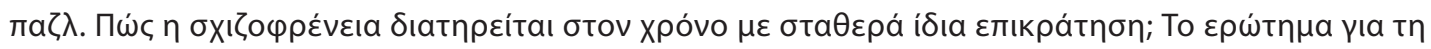

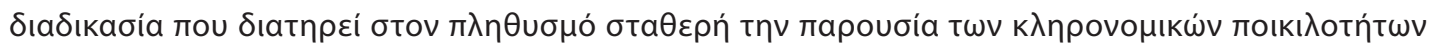

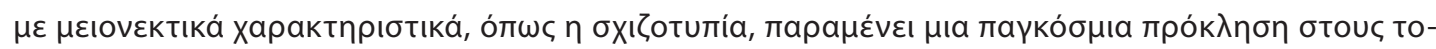

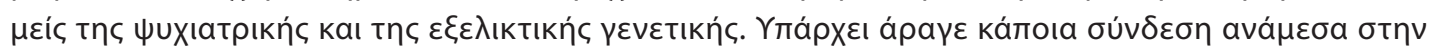

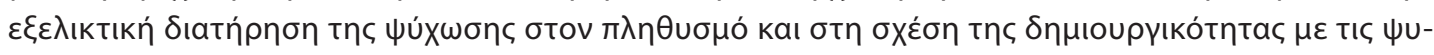
$\chi \omega \tau$ тко́

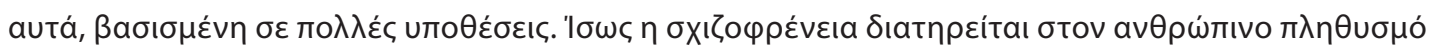

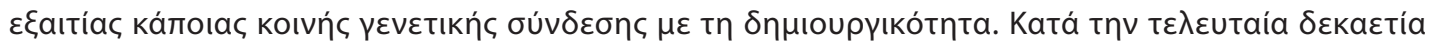

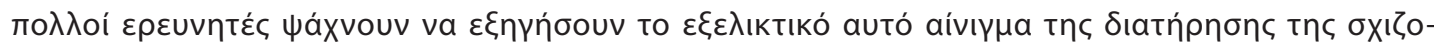

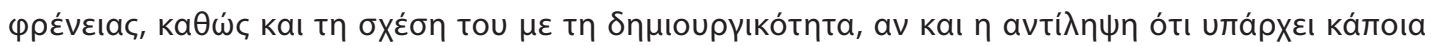

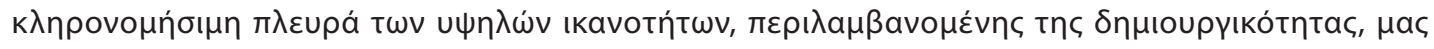

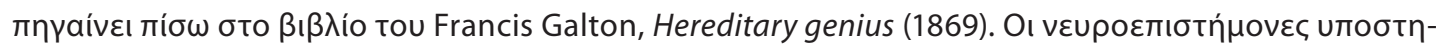




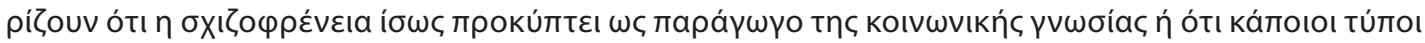

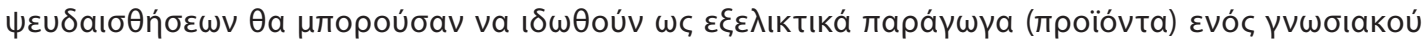

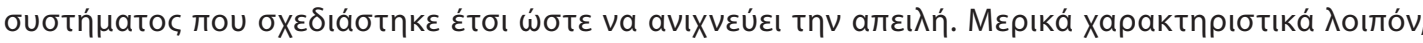

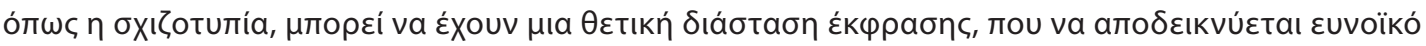

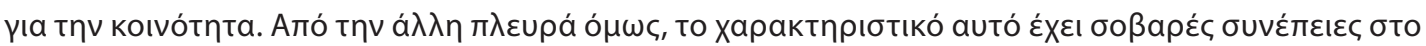

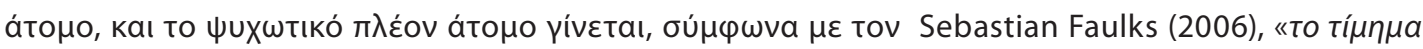

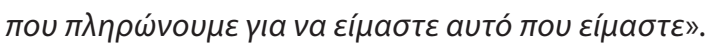

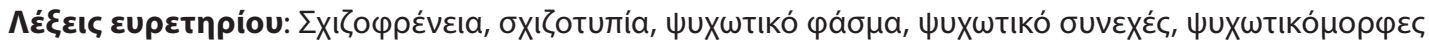

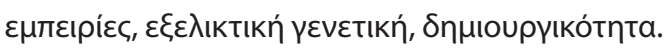

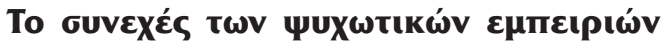

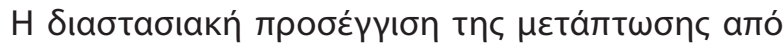

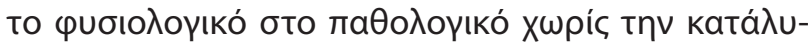

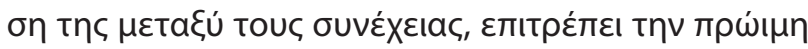

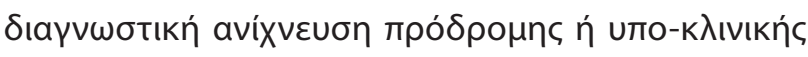

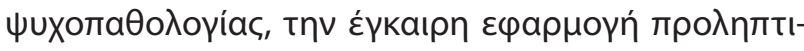

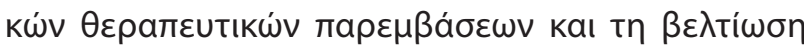

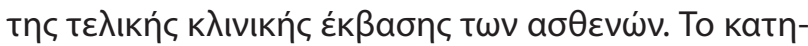

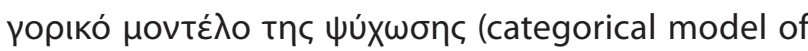

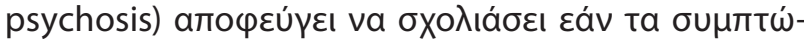

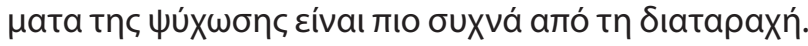

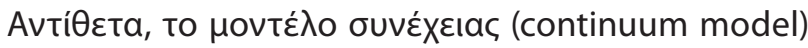

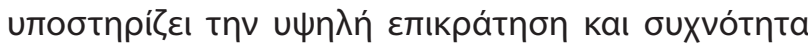

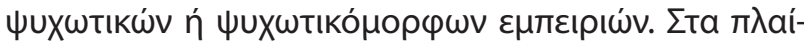

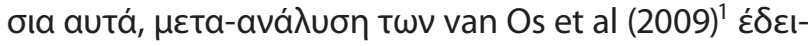

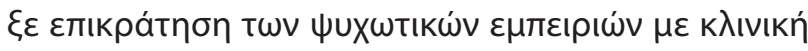

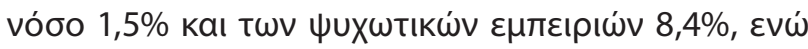

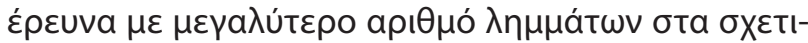

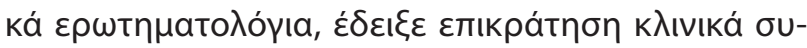

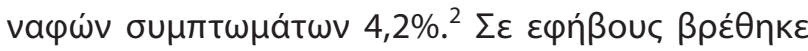

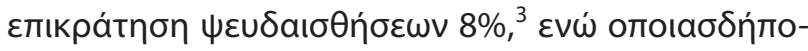

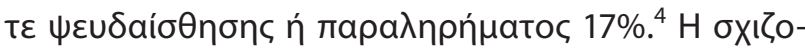

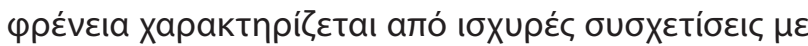

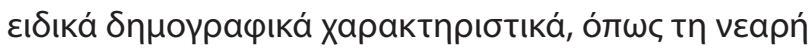

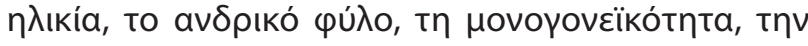

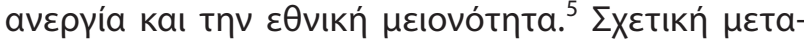

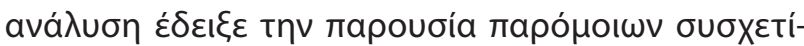

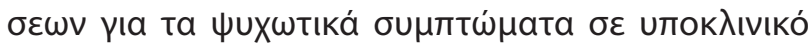

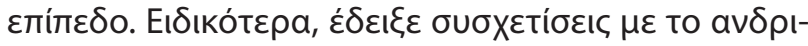

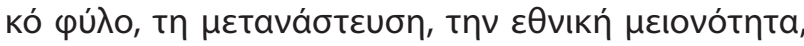

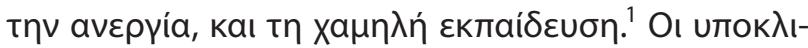

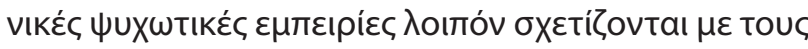

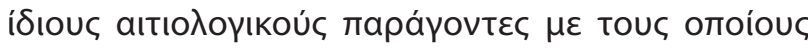

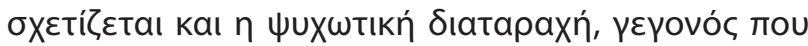

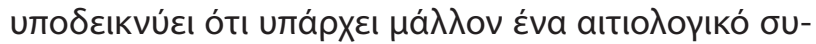

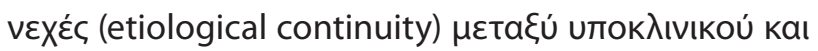

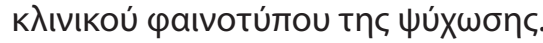

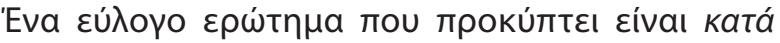

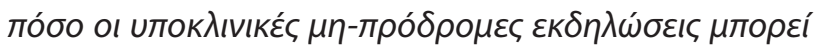

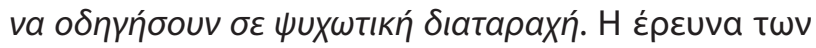

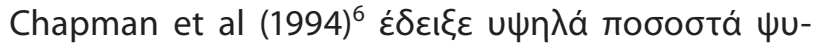

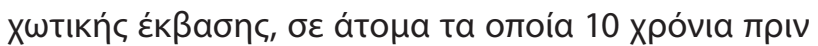

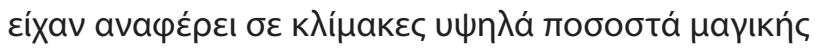

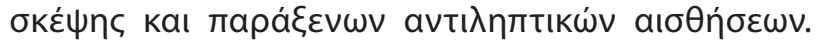

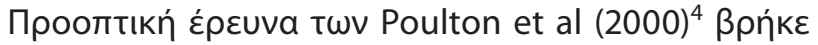

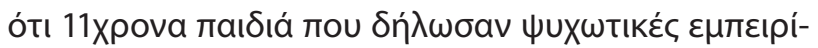

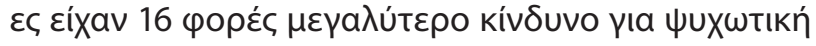

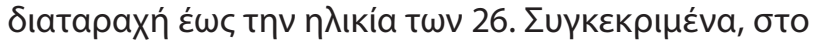

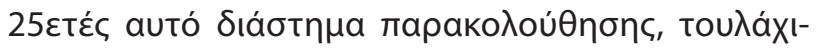

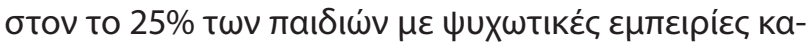

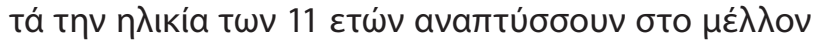

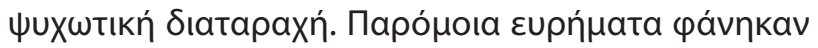

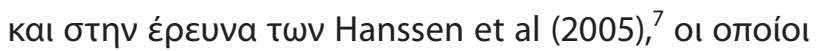

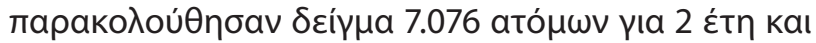

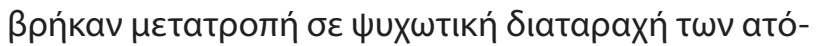

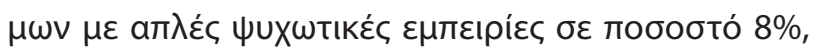

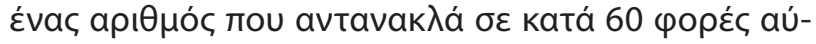

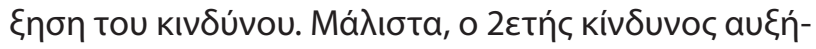

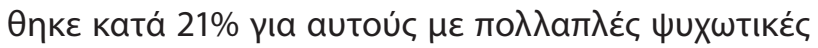

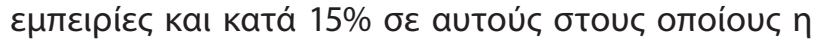

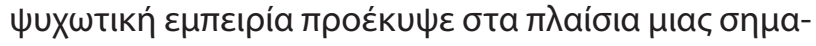

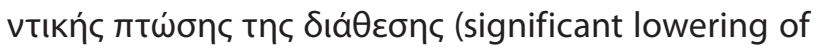

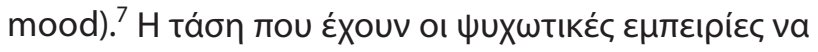

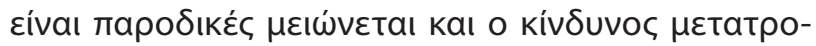

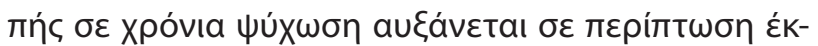

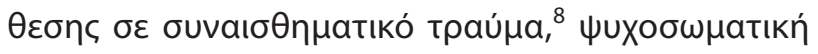

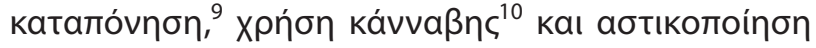
(urbanicity). ${ }^{8,11}$ 


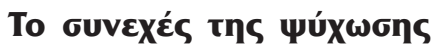

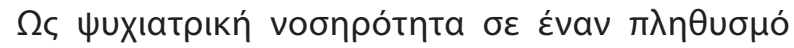

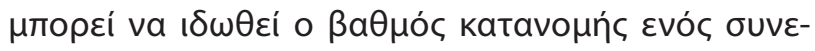

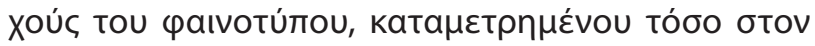

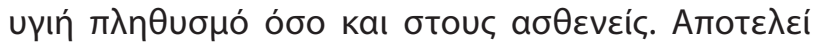

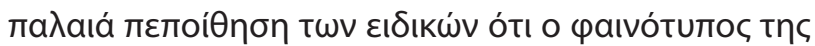

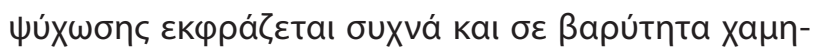

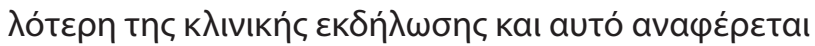

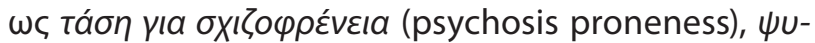
Х

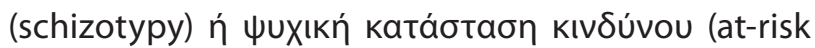

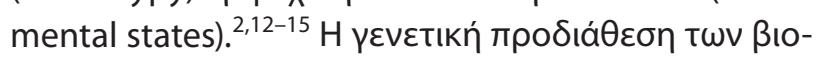

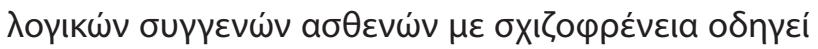

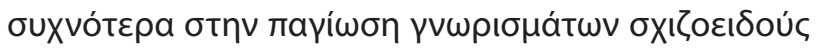

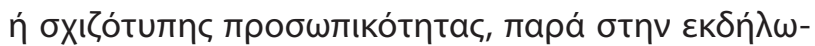

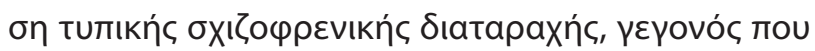

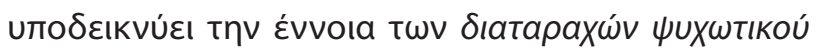

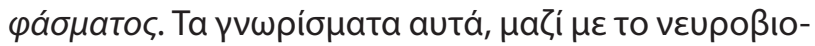

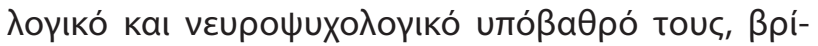

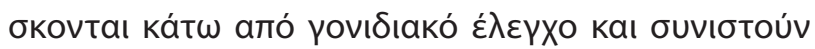

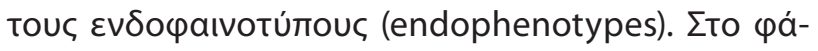

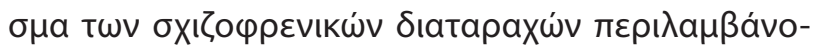

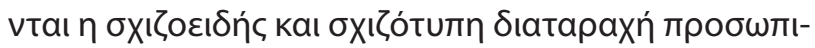

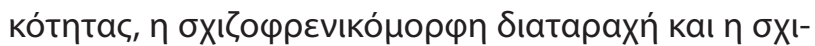

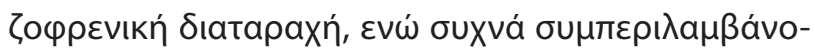

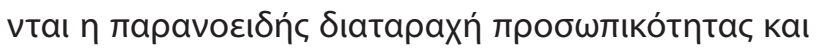

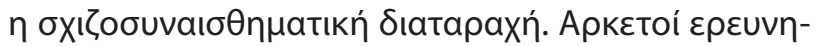

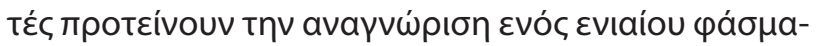

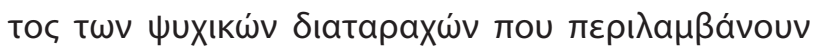

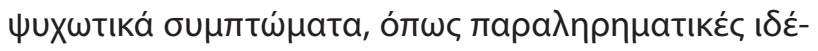

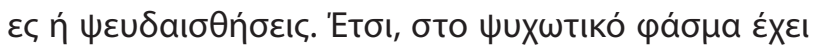

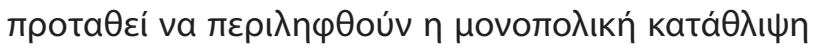

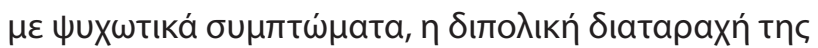

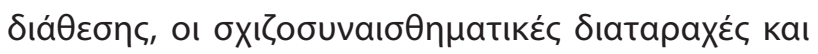

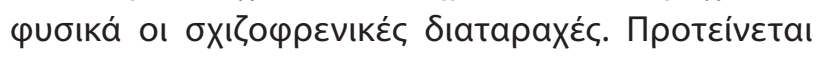

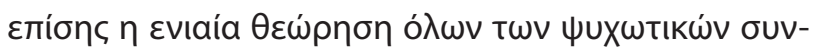

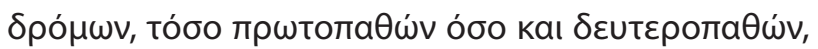

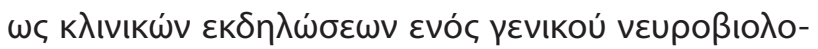

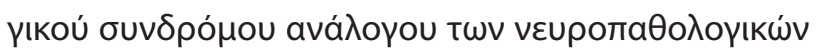

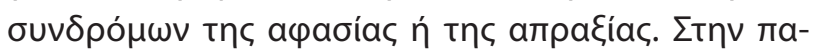

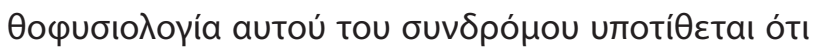

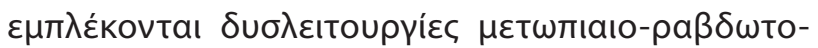

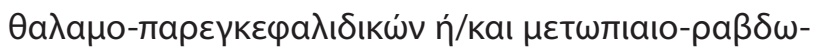

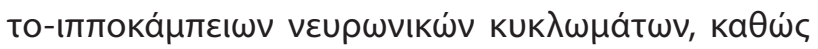
каı о проßо

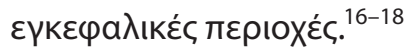

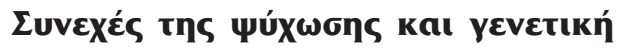

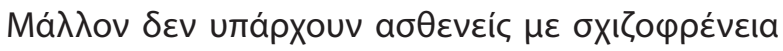

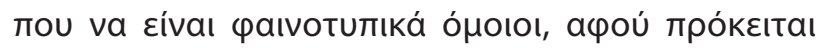

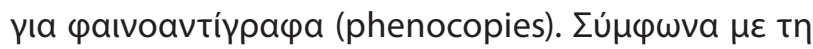

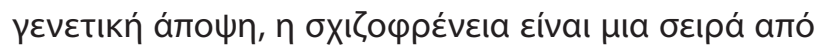

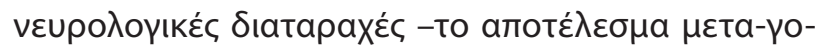

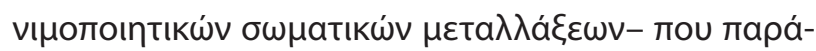

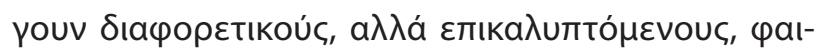

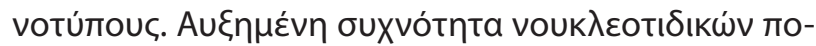

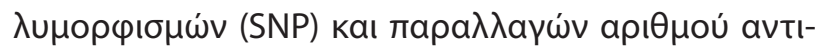

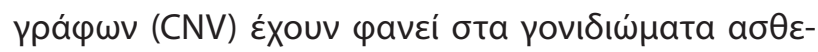

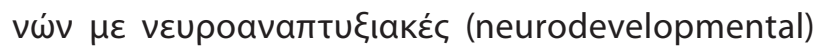

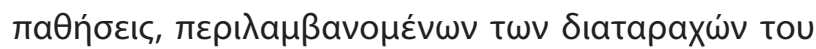

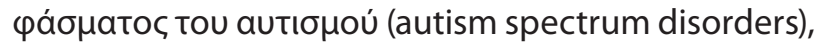

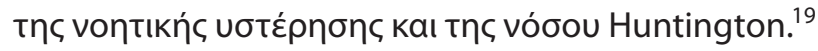

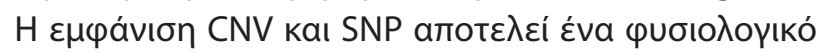

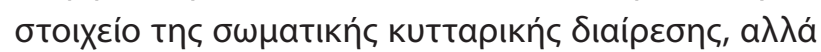

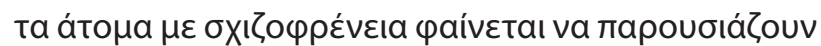

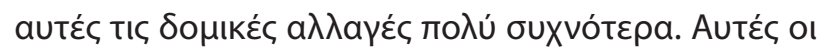

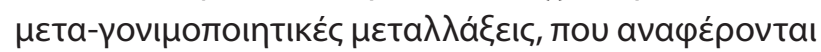

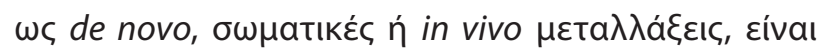

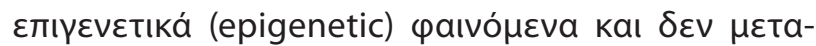

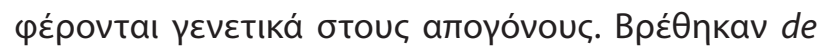

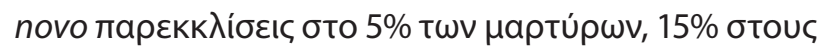

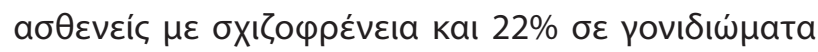

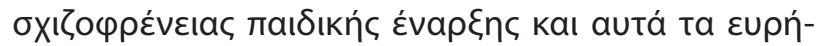

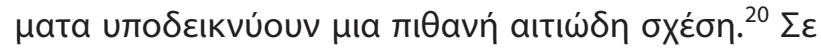

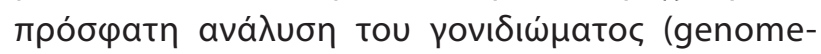

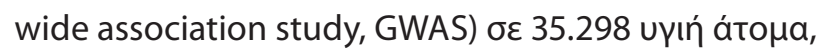

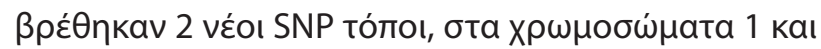

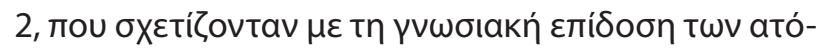

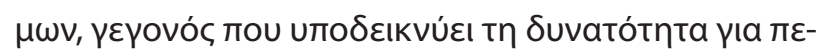

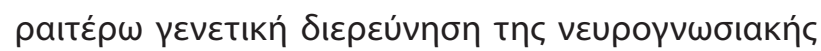

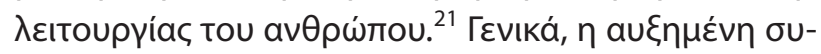

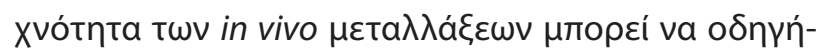

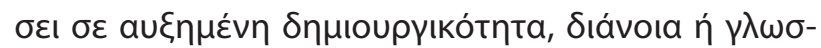

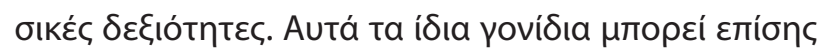

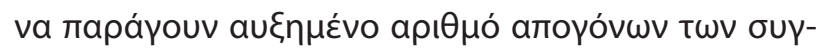

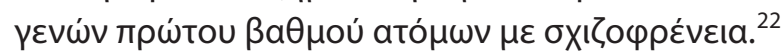

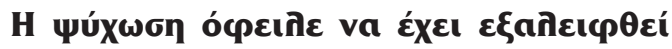

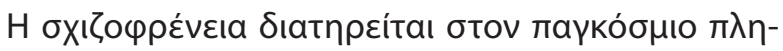

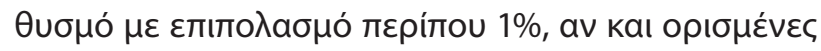

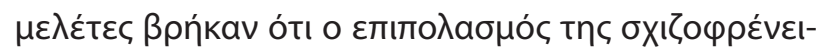

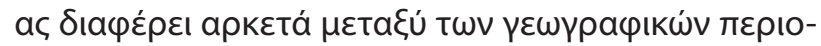




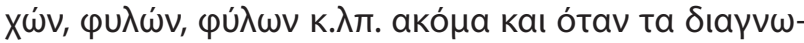

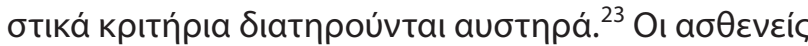

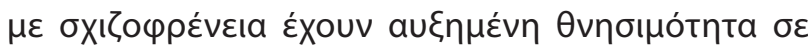

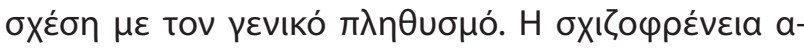

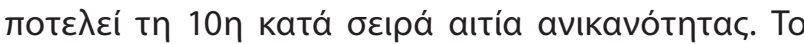

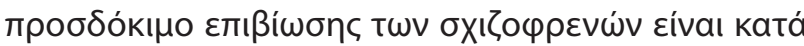

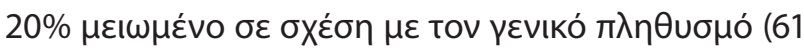

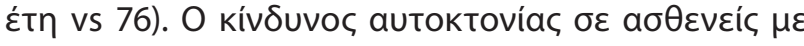

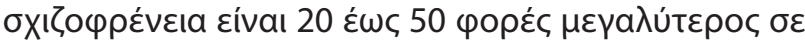

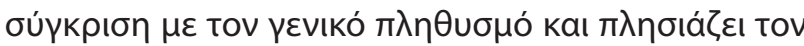

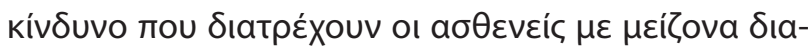

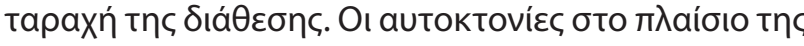

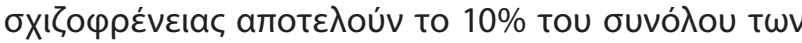

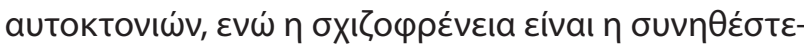

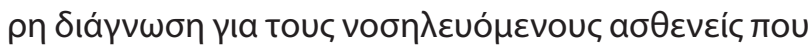
autokTovoúv. ${ }^{24}$

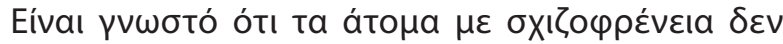

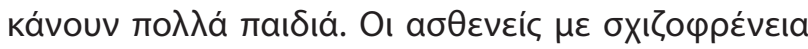

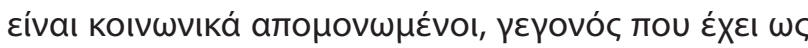

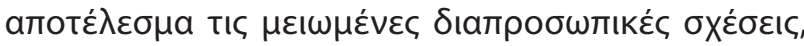

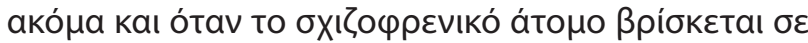

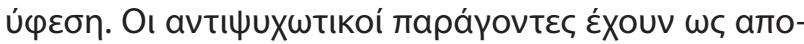

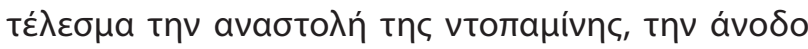

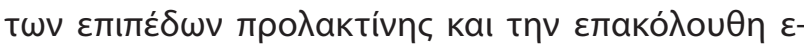

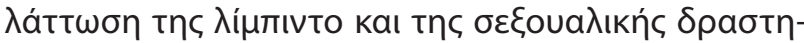

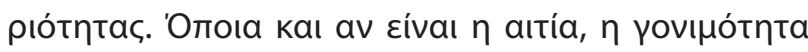

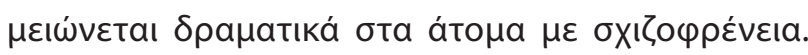

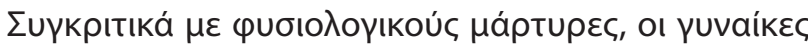

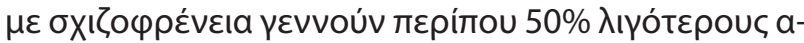

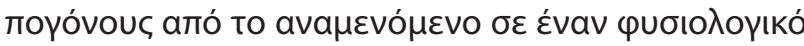

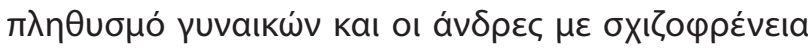

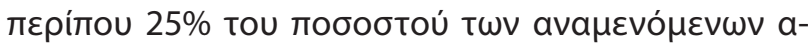

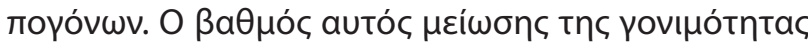

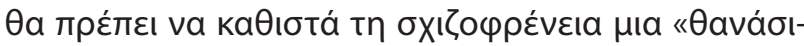

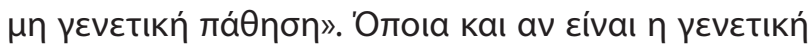

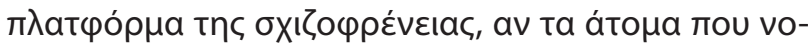

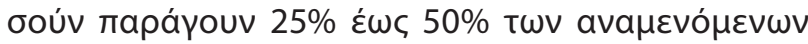

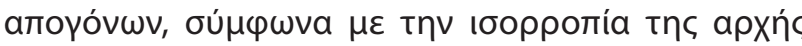

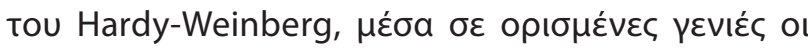

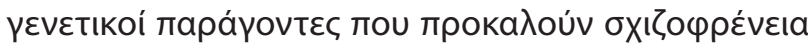

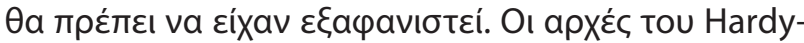

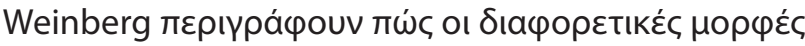

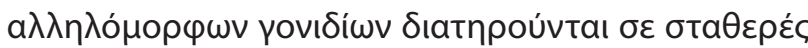

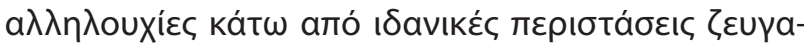

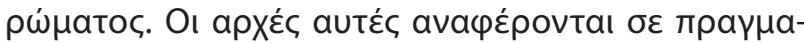

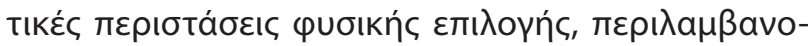

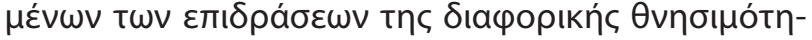

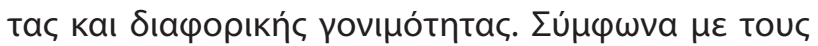

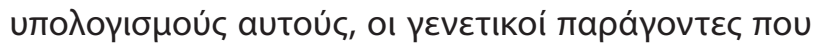

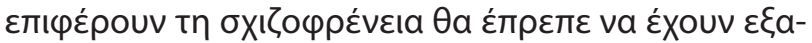

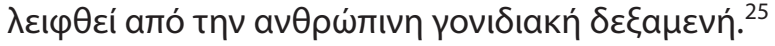

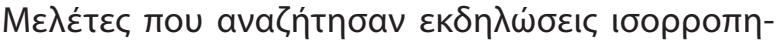

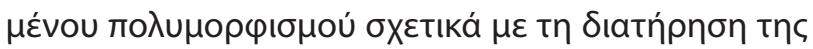

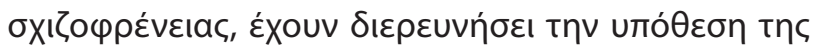

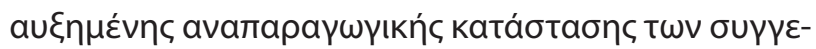

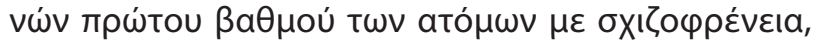

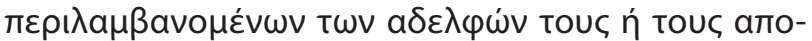

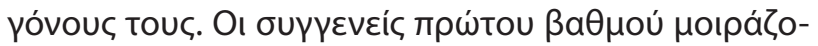

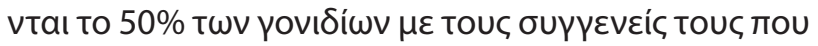

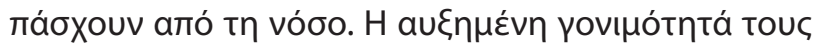

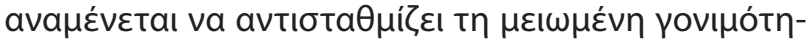

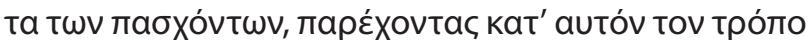

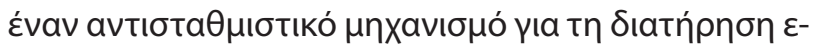

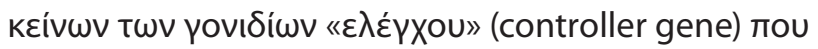

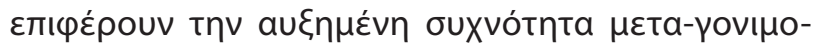

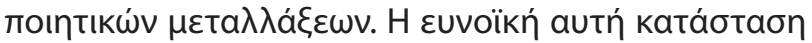

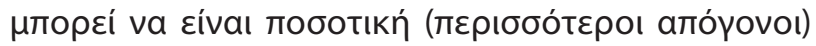

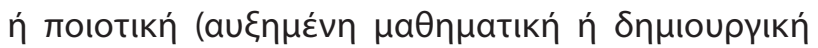

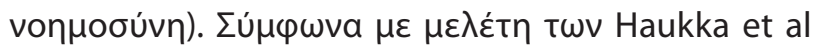

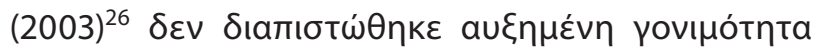

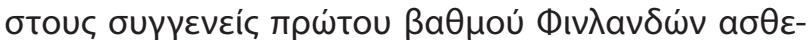

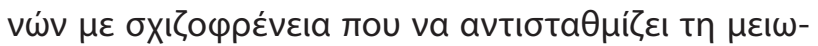

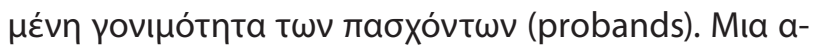

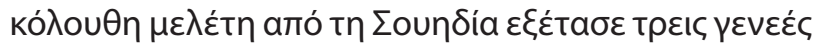

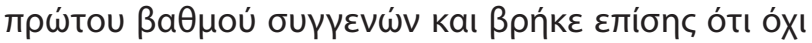

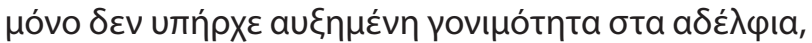

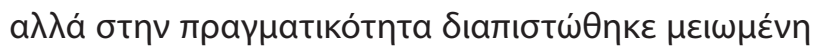

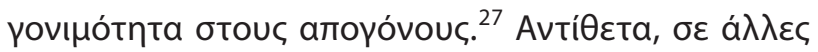

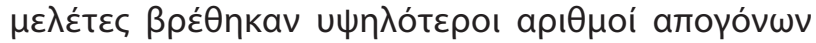

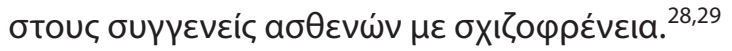

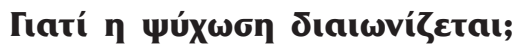

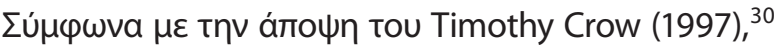

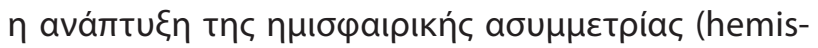

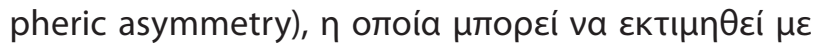

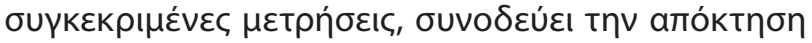

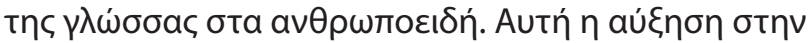

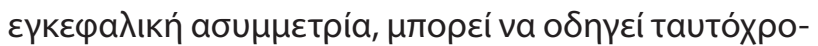

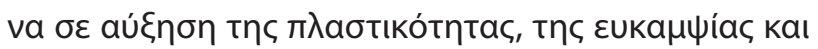

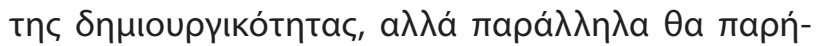

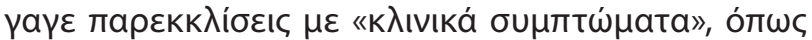

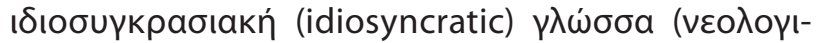




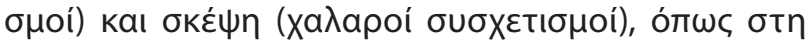

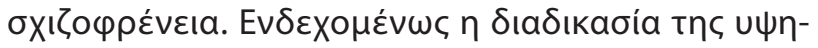

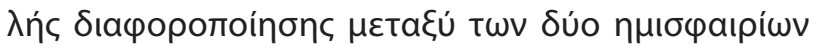

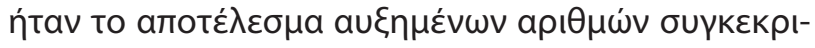

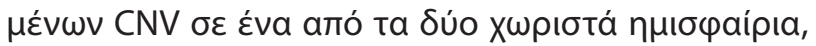

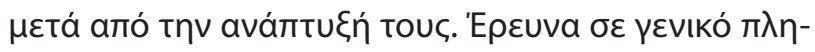

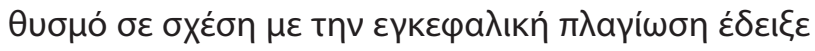

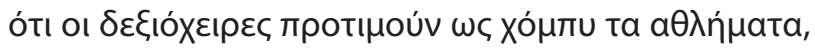

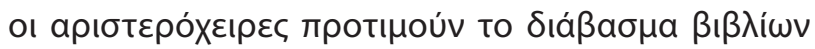

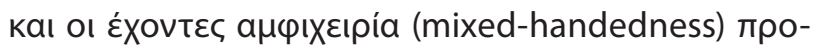

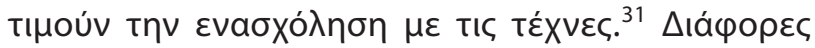

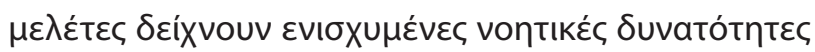

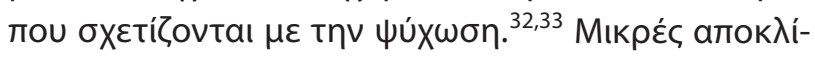

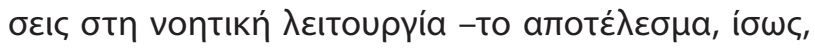

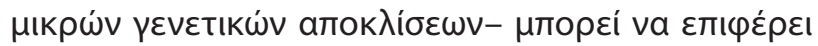

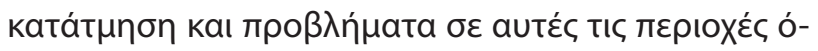

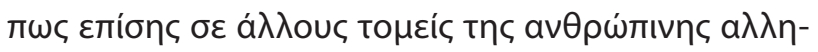
$\lambda \varepsilon \pi$ íSpaonc. ${ }^{34}$

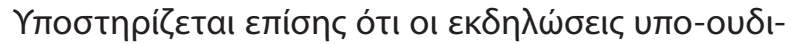

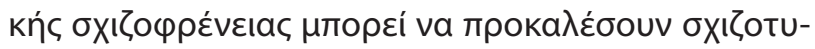

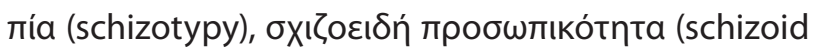

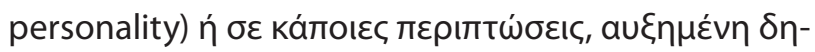

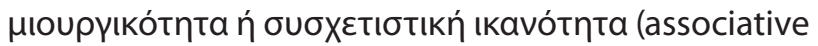

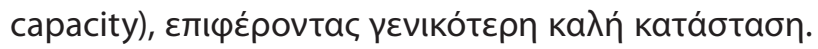

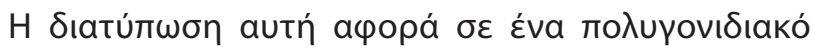

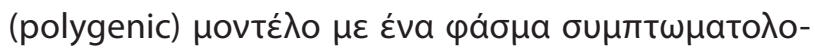

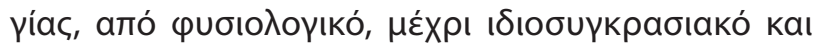

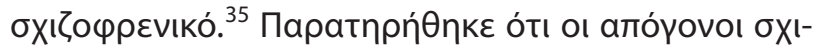

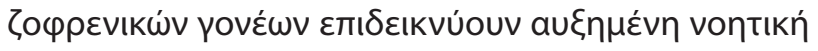

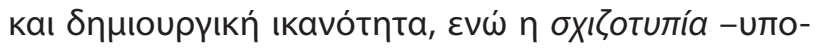

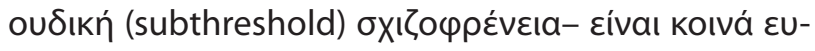

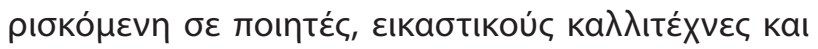

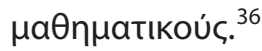

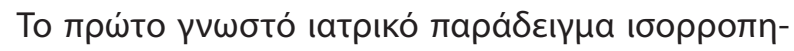

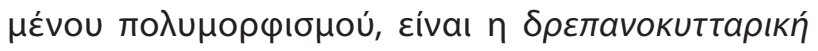

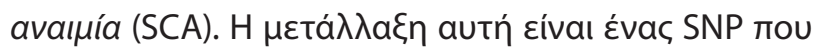

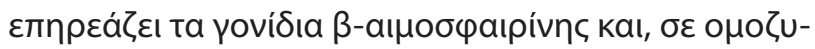

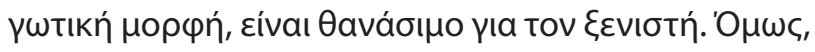

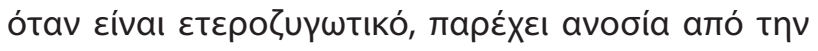

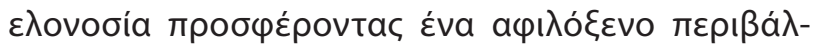
$\lambda$ ov yıa to Plasmodium falciparum, то парáбıто бто

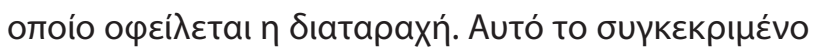

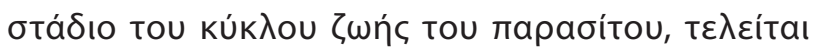

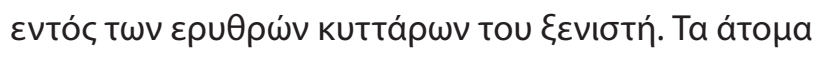

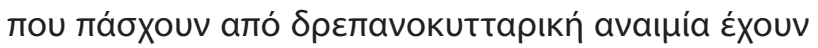

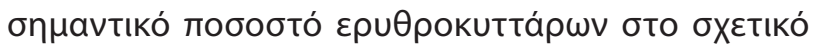

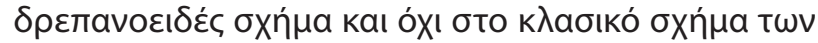

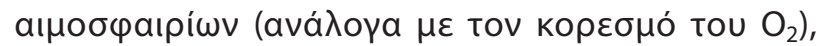

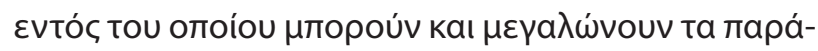

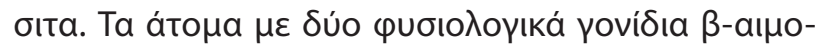

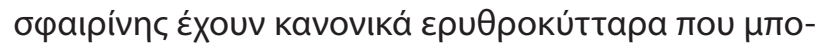

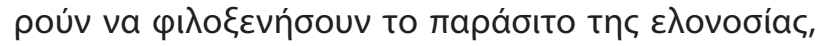

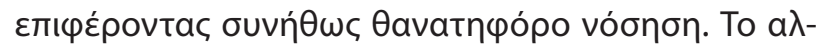

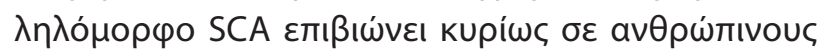

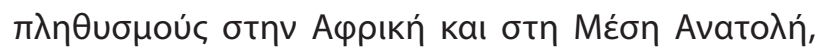

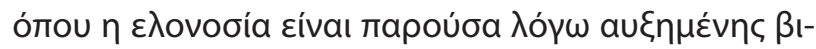

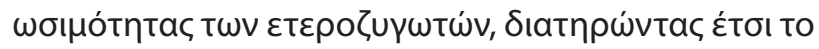

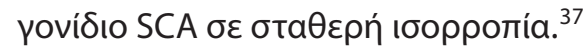

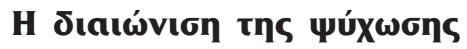

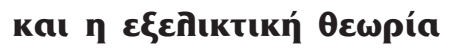

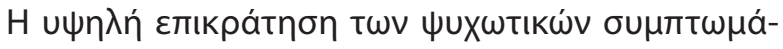

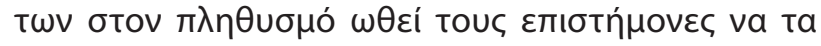

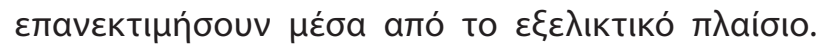

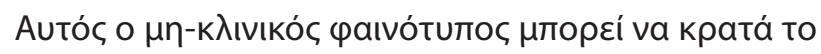
к $\lambda \varepsilon ı \delta$ tí

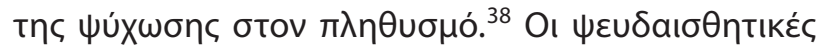

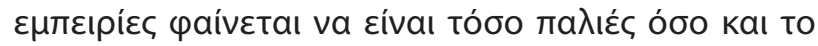

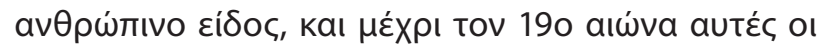

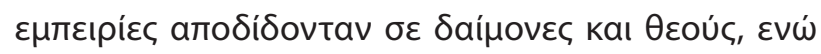

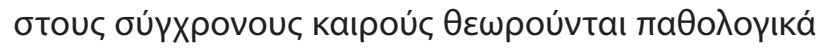

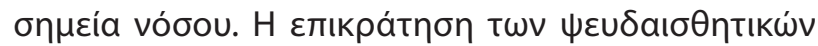

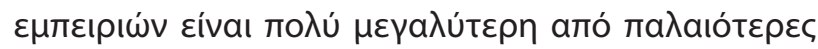

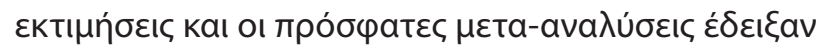

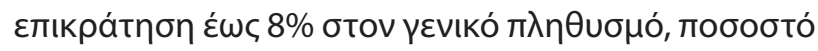

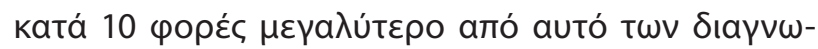

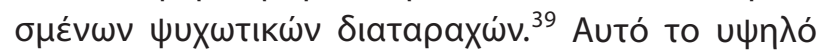

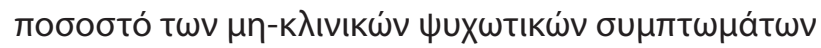

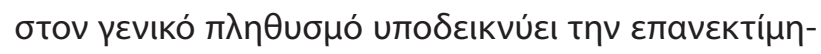

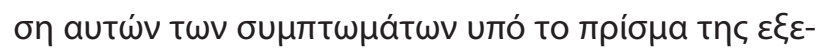
$\lambda$ Iктıкńc $\theta \varepsilon \omega$ píaç. ${ }^{40}$

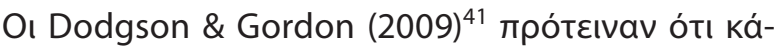

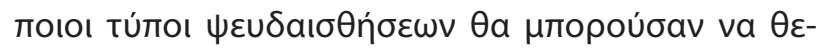

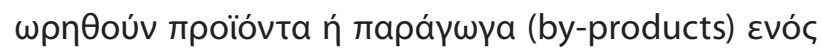

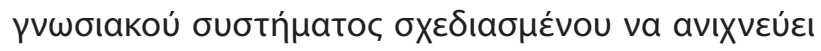

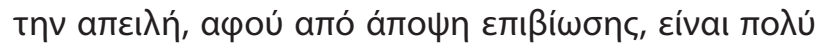

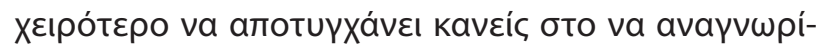

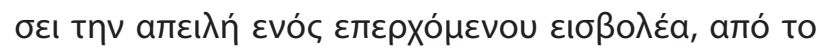

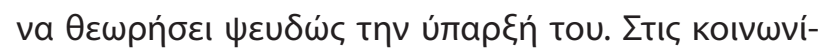

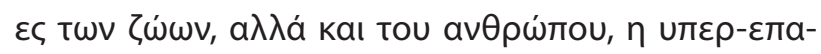

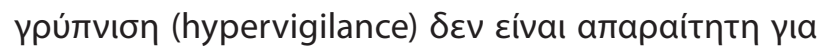

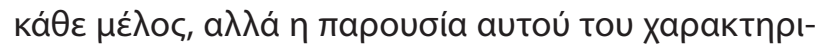




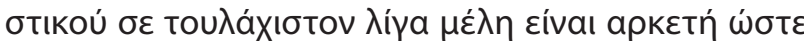

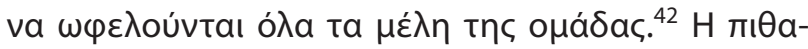

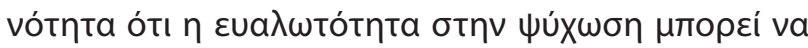

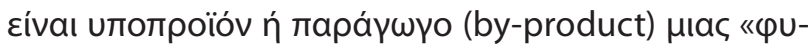

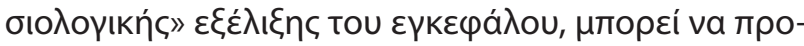

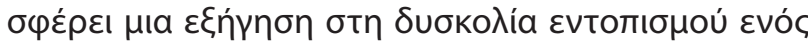

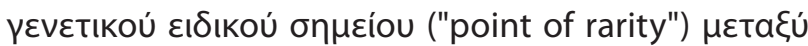

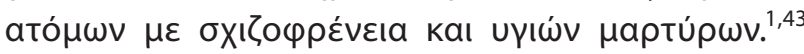

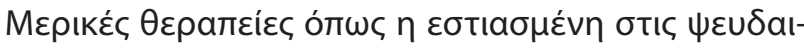

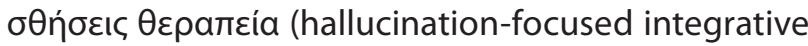

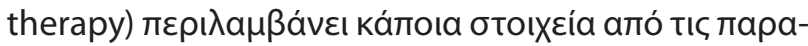

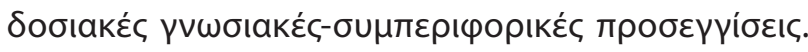

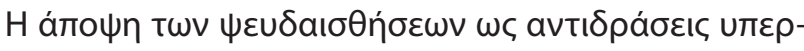

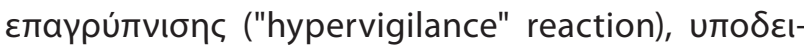

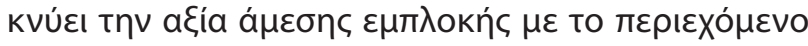

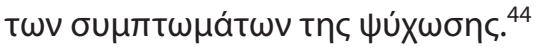

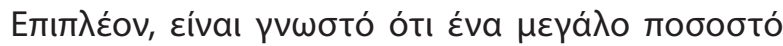

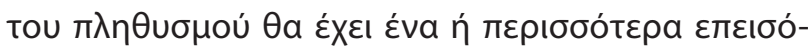

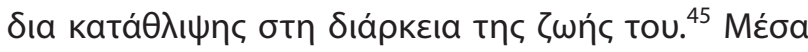

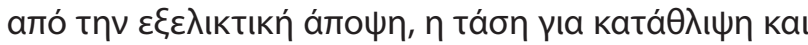

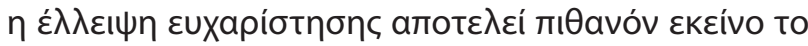

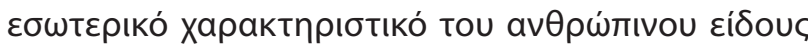

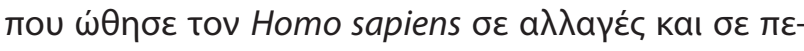

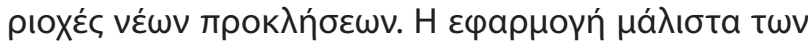

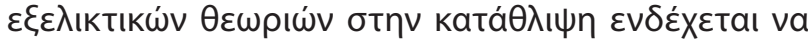
о

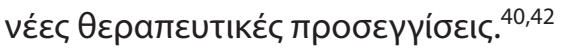

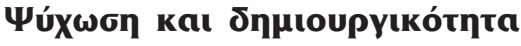

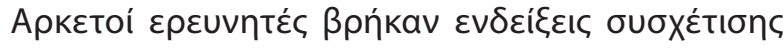

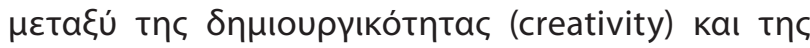

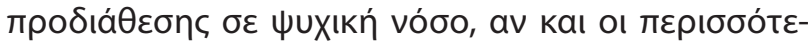

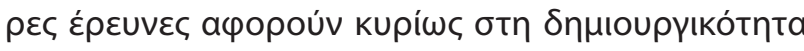

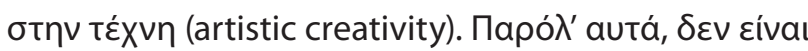

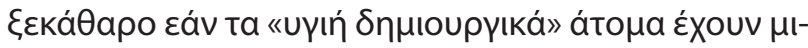

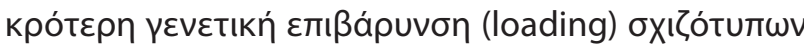

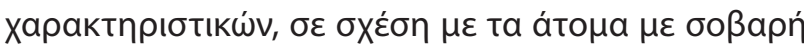

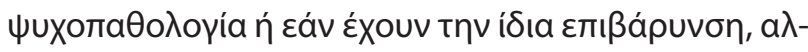

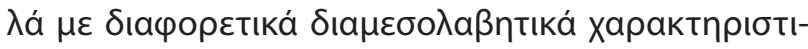

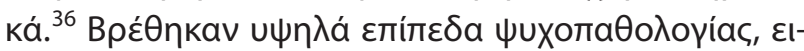

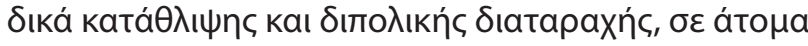

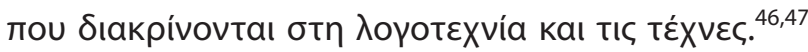

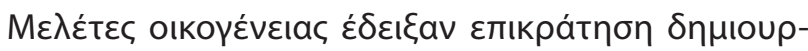

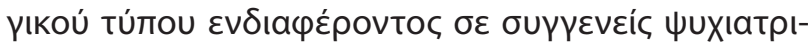

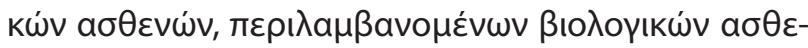

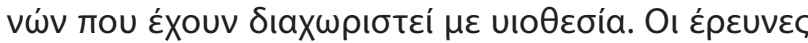

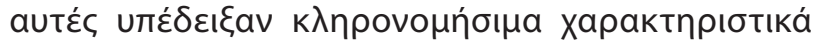

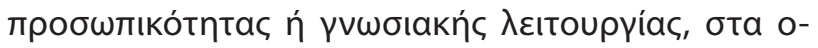

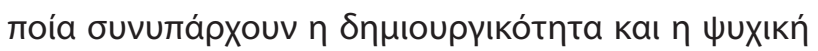

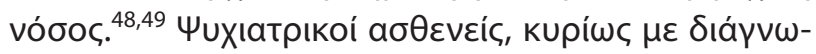

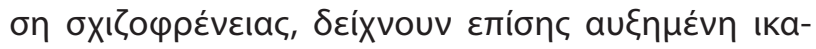

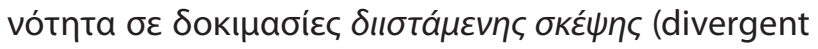

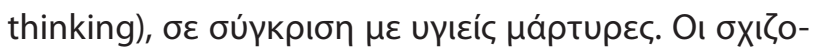

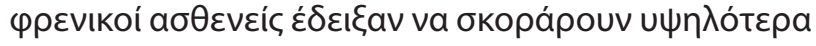

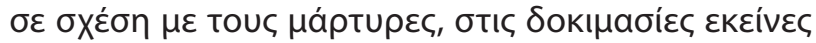

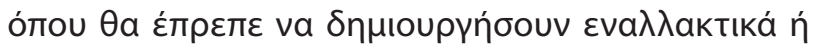

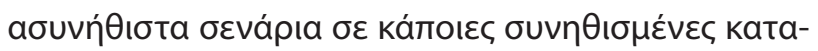

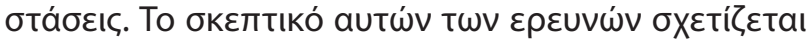

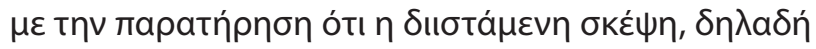

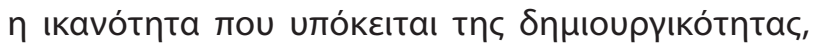

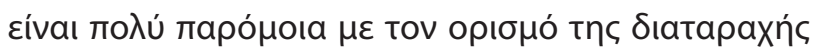

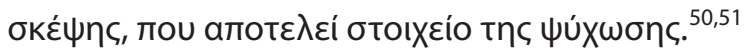

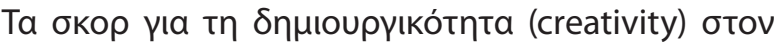

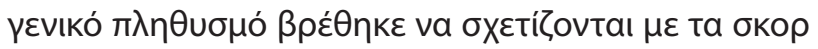

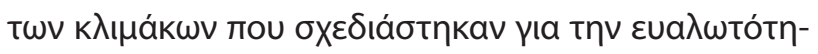

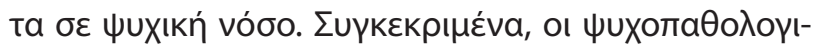

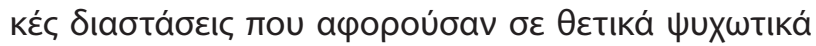

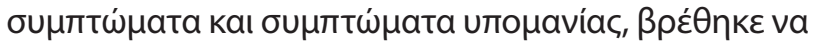

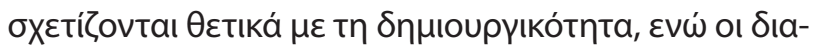

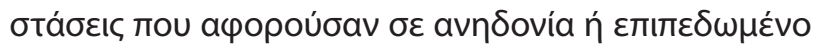

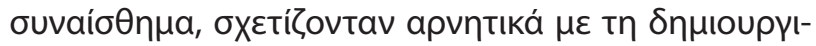

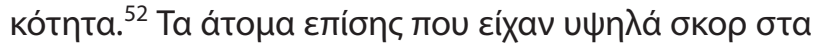

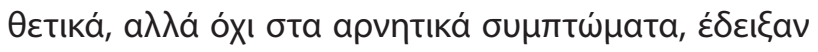

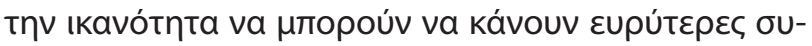

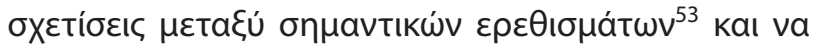

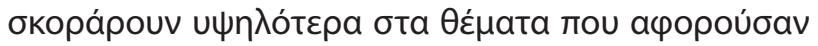

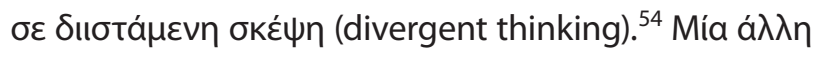

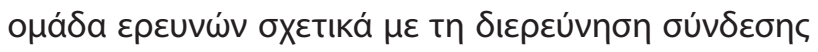

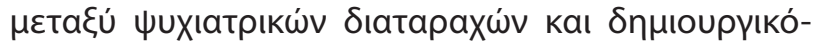

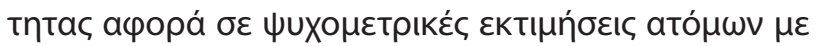

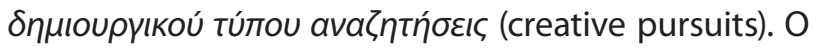

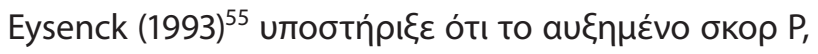

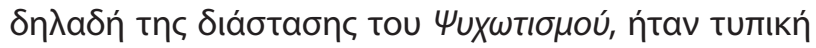

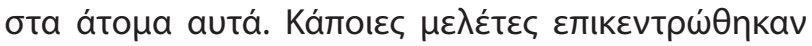

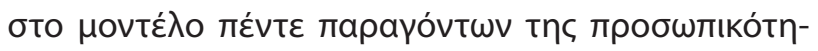
Tac (the five factor model of personality), то опоі́ $\delta \varepsilon v$

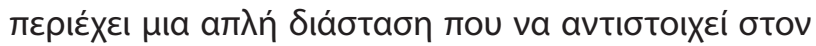

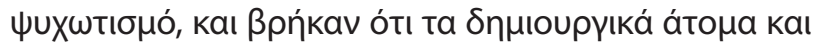

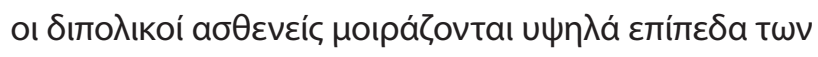

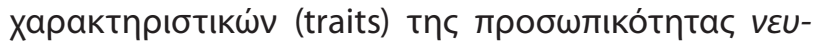

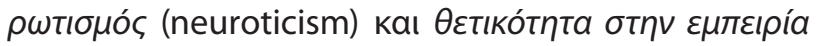

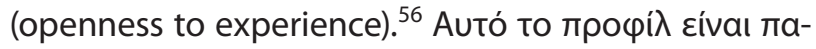




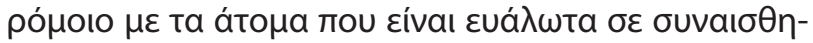

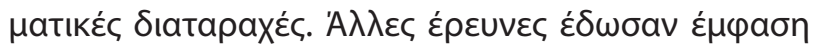

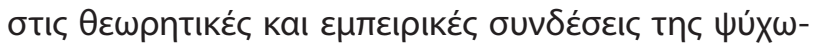

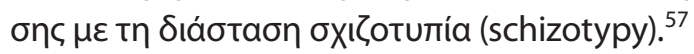

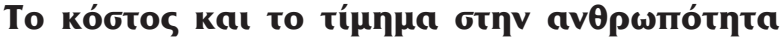

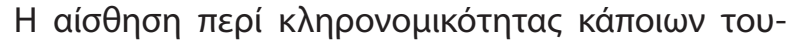

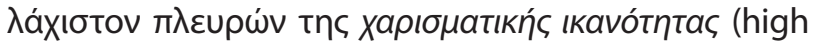

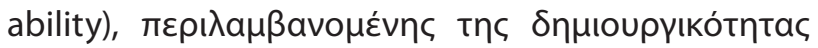

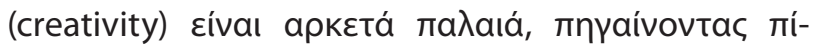
$\sigma \omega$ бто $\beta$ ı $\beta \lambda$ ío tou Francis Galton's Hereditary genius

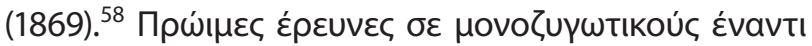

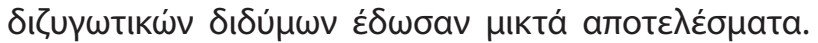

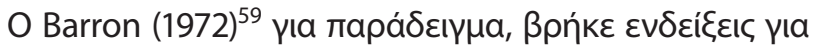

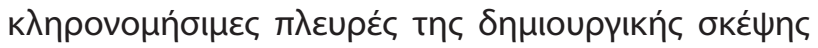

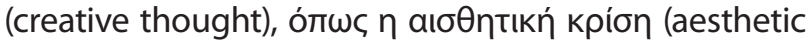

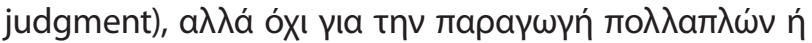

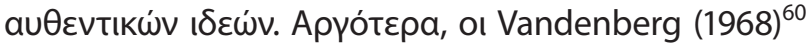

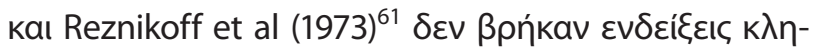

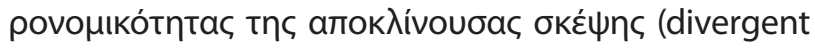

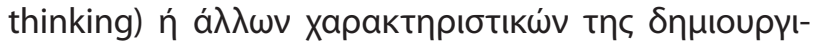

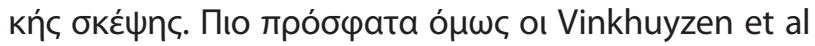

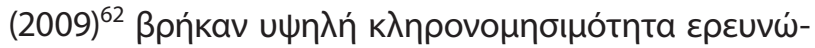

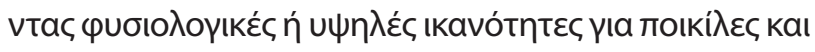

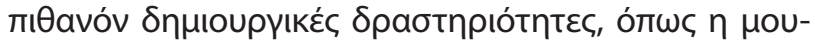

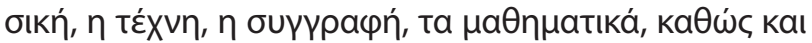

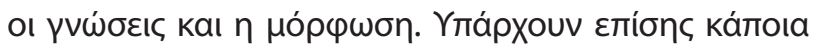

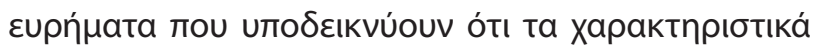

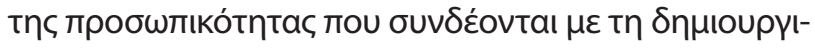

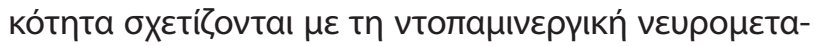

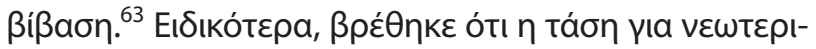

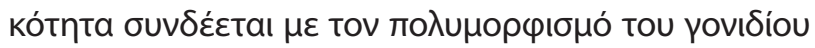

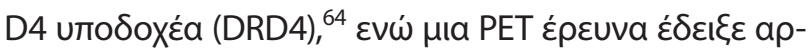

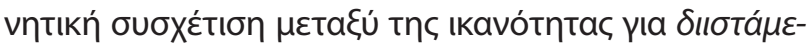

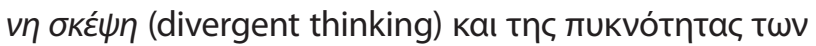

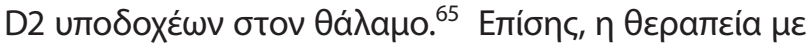

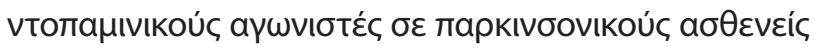

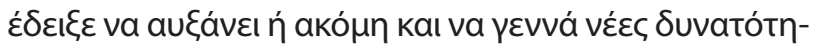

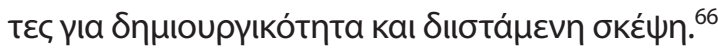

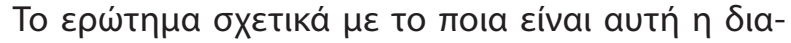

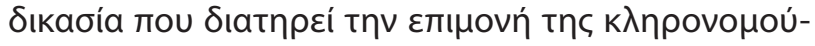

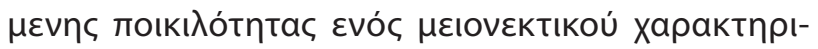

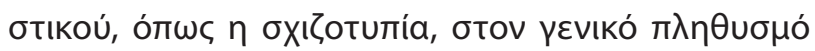

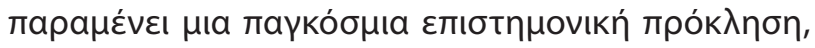

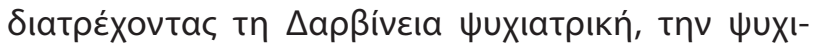

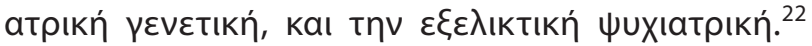

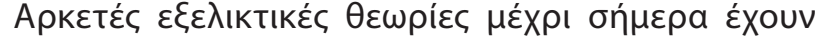

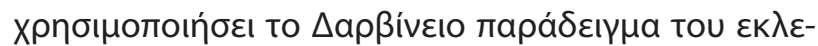

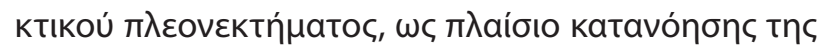

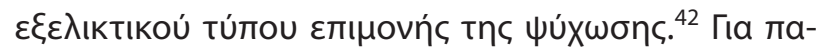

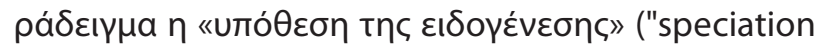

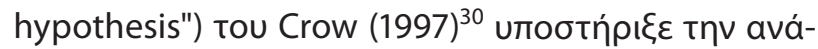

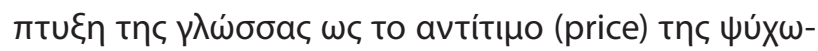

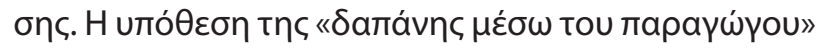

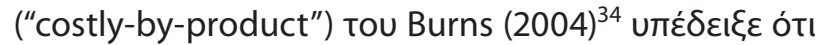

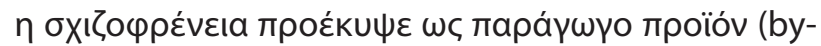

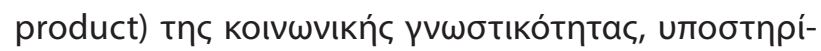

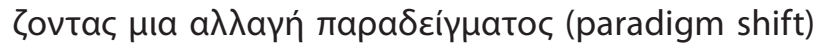

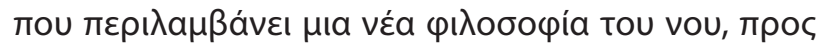

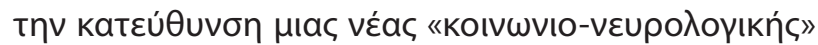

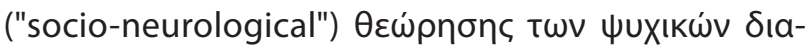

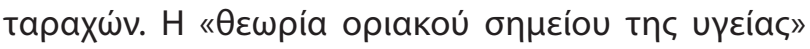

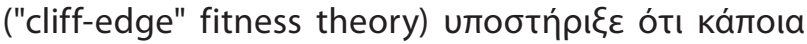

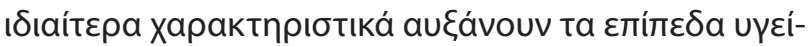

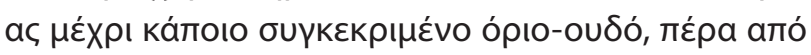

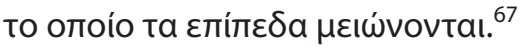

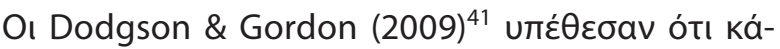

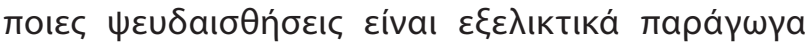

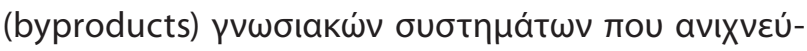

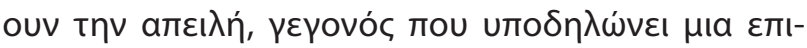

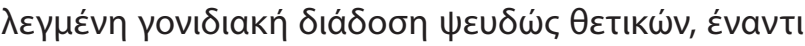

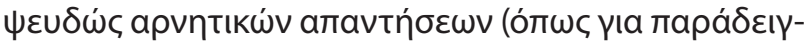

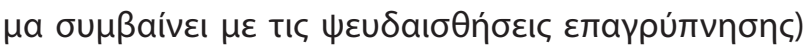

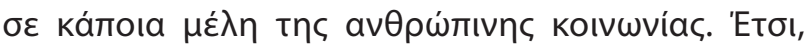

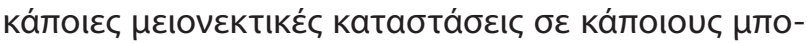

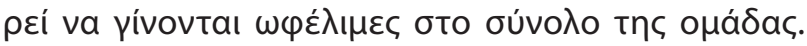
Oı Crespi \& Badcock (2008) ${ }^{68}$ kaı Del Giudice et al,

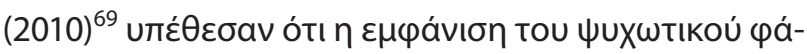

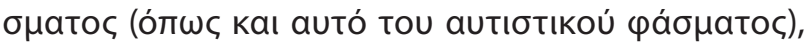

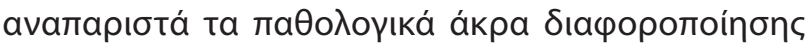

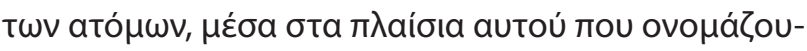

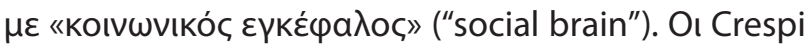

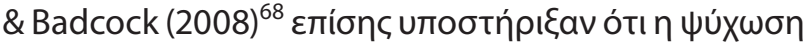

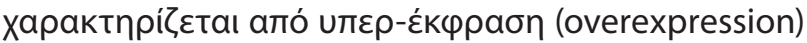
$\tau \omega v \varepsilon \kappa \varphi \rho a \zeta o ́ \mu \varepsilon v \omega v$ a

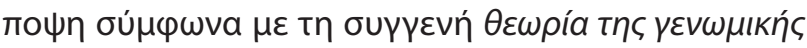

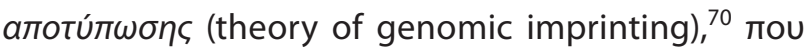

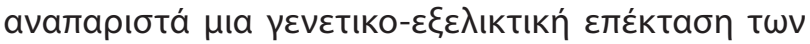

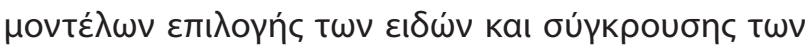

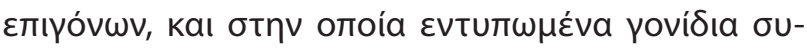

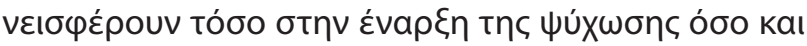

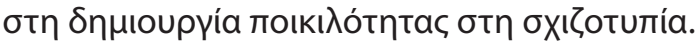




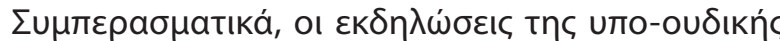

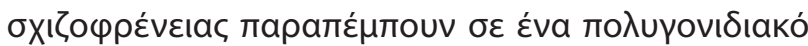

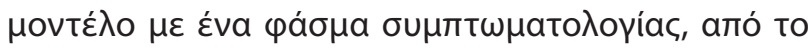

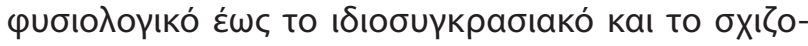

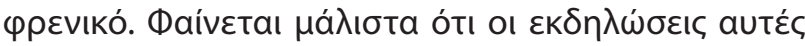

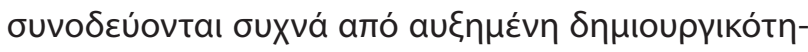

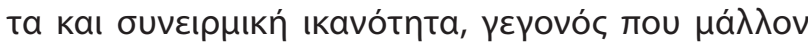

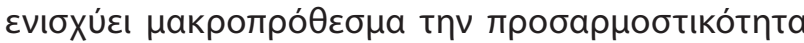

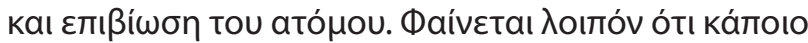

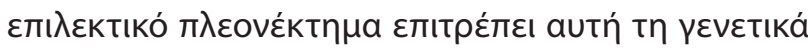

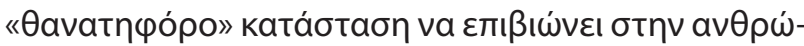

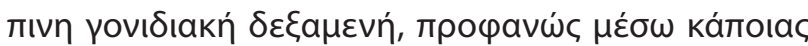

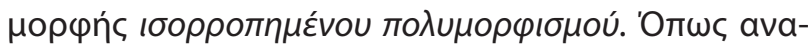
$\lambda$

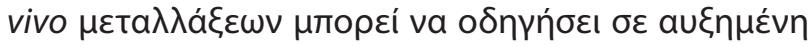

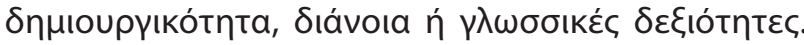

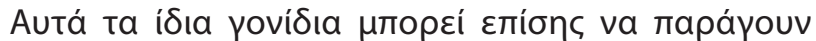

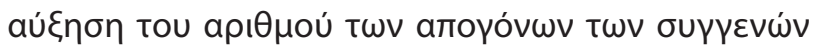

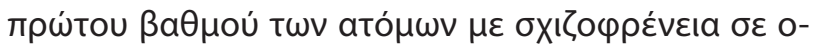

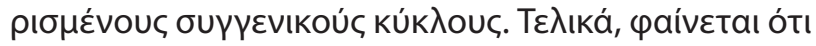

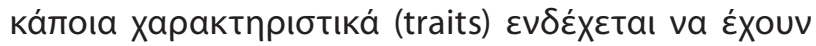

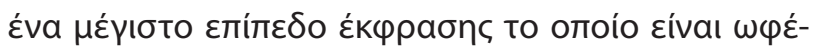

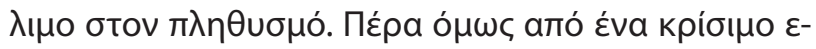

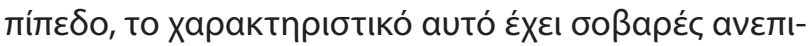
Өú

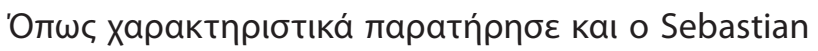

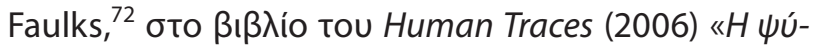

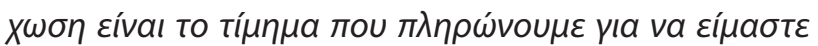

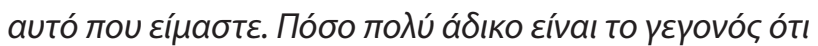

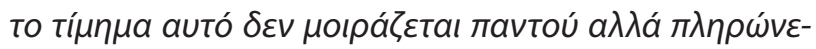

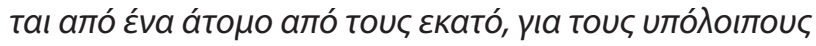

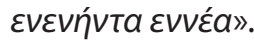

\title{
Persistence of psychosis in the population: The cost and the price for humanity
}

\author{
O. Giotakos
}

The Non-Profit Organization "Obrela", Athens, Greece

Psychiatriki 2018, 29:316-326

Schizophrenia is a chronic and highly impairing condition that affects around $1 \%$ of the human population. Evolutionary theories lend support for the idea of a continuum approach to the diagnosis of psychosis. Subclinical psychotic-like experiences are relatively common in children and adolescents, occurring in about $17 \%$ of youths. The prevalence rate of psychotic symptoms in the general population is up to $8 \%$, which is about ten times higher than the prevalence of diagnosed psychotic disorders. Some scientists have argued that there may be a shared genetic variation between illness and non-clinical psychotic-like symptom expression. The high prevalence of non-clinical psychotic symptoms in the population prompt neuroscientists to re-evaluate these symptoms in the light of evolutionary theory. The schizophrenia impaired physical health and reduced probability of reproduction raises an evolutionary puzzle. How does schizophrenia persist in the population at a stable prevalence rate? The question regarding what processes maintain the persistence of high heritable variation in relatively disadvantageous traits, such as schizotypy, in the general population, remains a universal challenge across the domains of psychiatric and evolutionary genetics. Furthermore, is there any link between the evolutionary persistence of psychosis in the population worldwide and the association of creativity with psychoticlike experiences in the general population? There has been a healthy debate on many hypotheses. One possibility is that schizophrenia remains in the human population because of shared genetic linkages to creativity. During last decade many scientists search the explanation for the evolutionary enigma of the persistence of schizophrenia, and the nature of its relation to creativity, although the notion that there is a heritable aspect of at least some aspects of high ability, including creativity, is going back at least to 
Francis Galton's book Hereditary genius (1869). Neuroscientists also suggested that schizophrenia may emerge as a by-product of social cognition or that certain types of hallucinations could be viewed as evolutionary by-products of a cognitive system designed to detect threat. Some traits, such as schizotypy, may have an optimal level of expression that is advantageous within the community. However, this trait has adverse consequences for the psychotic person, which becomes, as Sebastian Faulks (2006) suggested, "the price we pay for being what we are".

Key words: Schizophrenia, schizotypy, psychosis spectrum, psychosis continuum, psychotic-like experiences, evolutionary genetics, creativity.

\section{Bıßהtoypacpía}

1. van Os J, Linscott R, Myin-Germeys J, Delespaul P, Krabbendam L. A systematic review and meta-analysis of the psychosis continuum: evidence for a psychosis proneness-persistenceimpairment model of psychotic disorder. Psychol Med 2009, 39, 179-195, doi: 10.1017/S0033291708003814

2. van Os J. A salience dysregulation syndrome. Br J Psychiatry 2009, 194: 101-103, doi: 10.1192/bjp.bp. 108.054254

3. McGee R, Williams S, Poulton R. Hallucinations in nonpsychotic children. J Am Acad Child Adolesc Psychiatry 2000, 39:12-13, doi: 10.1097/00004583-200009000-00002

4. Poulton R, Caspi A, Moffitt TE, Cannon M, Murray R, Harrington $H$. Children's self-reported psychotic symptoms and adult schizophreniform disorder: a 15-year longitudinal study. Arch Gen Psychiatry 2000, 57:1053-1058, doi:10.1001/archpsyc. 57.11.1053

5. McGrath J, Saha S, Welham J, El Saadi O, MacCauley C, Chant D. A systematic review of the incidence of schizophrenia: the distribution of rates and the influence of sex, urbanicity, migrant status and methodology. BMC Med 2004, 2:13, doi: 10.1186/1741-7015-2-13

6. Chapman JP, Chapman LJ, Kwapil TR. Does the Eysenck Psychoticism dimension predict psychosis? A ten year longitudinal study. Pers Individ Dif 1994, 17:369-375, doi: 10.1016/ 0191-8869(94)90284-4

7. Hanssen M, Bak M, Bijl R, Vollebergh W, van Os J. The incidence and outcome of subclinical psychotic experiences in the general population. Br J Clin Psychol 2005, 44:181-191, doi: 10.1348/014466505X29611

8. Spauwen J, Krabbendam L, Lieb R, Wittchen HU, van Os J. Evidence that the outcome of developmental expression of psychosis is worse for adolescents growing up in an urban environment. Psychol Med 2006, 36: 407-415, doi:10.1017/ S0033291705006902

9. Dimitrakopoulos S, Vitoratou S, Mougiakos T, Bogeas N, Giotakos O, van Os J et al. Steinberg and Durell (1968) revisited: increased rates of First Episode Psychosis following military induction in Greek Army Recruits. Psychol Med 2017, 22:1-9, doi: $10.1017 /$ S0033291717002276.

10. Henquet $\mathrm{C}$, Murray $\mathrm{R}$, Linszen $\mathrm{D}$, van Os $\mathrm{J}$. The environment and schizophrenia: the role of cannabis use. Schizophr Bull 2005, 31:608-612, doi: 10.1093/schbul/sbi027
11. Spauwen J, Krabbendam L, Lieb R, Wittchen HU, van Os J. Does urbanicity shift the population expression of psychosis? J Psychiatr Res 2004, 38: 613-618, doi: 10.1016/j.jpsychires.2004.04.003

12. Stefanis NC, Delespaul P, Smyrnis N, Lembesi A, Avramopoulos DA, Evdokimidis IK et al. Is the excess risk of psychosis-like experiences in urban areas attributable to altered cognitive development? Soc Psychiatry Psychiatr Epidemiol 2004, 39: 364-368, doi: 10.1007/s00127-004-0771-3

13. Stefanis NC, Hanssen M, Smirnis NK, Avramopoulos DA, Evdokimidis IK, Stefanis CN et al. Evidence that three dimensions of psychosis have a distribution in the general population. Psychol Med 2002, 32: 347-358, doi: 10.1017\}S0033291701005141

14. Vollema MG, Sitskoorn MM, Appels MC, Kahn RS. Does the Schizotypal Personality Questionnaire reflect the biologicalgenetic vulnerability to schizophrenia? Schizophr Research 2002, 54:39-45, PMID: 11853977

15. Yung AR, Phillips LJ, Yuen HP, Francey SM, McFarlane CA, Hallgren $\mathrm{M}$ et al. Psychosis prediction: 12-month follow up of a high-risk ("prodromal") group. Schizophr Res 2003, 60: 21-32. PMID: 12505135

16. Fujii DE, Ahmed I (eds) The spectrum of psychotic disorders: neurobiology, etiology and pathogenesis. Cambridge, Cambridge University Press, 2007, doi: 10.1176/ps.2008.59.1.118

17. Giotakos O. Poor insight and psychosis. Psychiatriki 2017, 28 : 332-341, doi: 10.22365/jpsych.2017.284.332

18. Giotakos $\mathrm{O}$. Poor insight in psychosis and meta-representation models. Dialog J Clin Neurosci 2018, 1:12-25, doi: 10.26386/ obrela.v1i1.5

19. Flavell SW, Greenberg ME. Signaling mechanisms linking neuronal activity to gene expression and plasticity of the nervous system. Annu Rev Neurosci 2008, 31:563-590, doi: 10.1146/ annurev. neuro.31.060407.125631

20. Walsh T, McClellan JM, McCarthy SE, Addington AM, Pierce $\mathrm{SB}$, Cooper GM et al. Rare structural variants disrupt multiple genes in neurodevelopmental pathways in schizophrenia. Science 2008, 25, 320:539-543, doi: 10.1126/science.1155174

21. Trampush JW, Yang ML, Yu J, Knowles E4, Davies G, Liewald DC et al, GWAS meta-analysis reveals novel loci and genetic correlates for general cognitive function: a report from the COGENT consortium. Mol Psychiatry 2017, 22:336-345, doi: $10.1038 / \mathrm{mp} .2016 .244$ 
22. Keller M, Miller GF. An evolutionary framework for mental disorders: Integrating adaptationist and evolutionary genetics models. Behav Brain Sci 2006, 29:429-452, doi: 10.1017/ S0140525X06459094

23. Goldner EM, Hsu L, Waraich P, Somers JM. Prevalence and incidence studies of schizophrenic disorders: a systematic review of the literature. Can J Psychiatry 2002, 47:833-843, doi: $10.1177 / 070674370204700904$

24. Cardno A, Rijsdijk F, Sham P et al. A twin study of genetic relationships between psychotic symptoms. Am J Psychiatry 2002, 159:539-545, doi: 10.1176/appi.ajp.159.4.539

25. Bassett AS, Bury A, Hodgkinson KA, Honer WG. Reproductive fitness in familial schizophrenia. Schizophr Res 1996, 21:151160, PMID: 8885043

26. Haukka J, Suvisaari J, Lonnqvist J. Fertility of patients with schizophrenia, their siblings, and the general population: a cohort study from 1950 to 1959 in Finland. Am J Psychiatry 2003, 160:460-463, doi: 10.1176/appi.ajp.160.3.460

27. Svensson AC, Liechtenstein P, Sundin S, Hultman CM. Fertility of first-degree relatives of patients with schizophrenia; a three generation perspective. Schizophr Res 2007, 91:238-245, doi: 10.1016/j.schres.2006.12.002

28. Avila M, Thaker G, Adami H. Genetic epidemiology and schizophrenia: a study of reproductive fitness. Schizophr Res 2001, 47:233-241, PMID: 11278140

29. Weiser M, Reichenberg A, Werbeloff $\mathrm{N}$ et al. Increased number of offspring in first degree relatives of psychotic individuals: a partial explanation for the persistence of psychotic illnesses. Acta Psychiatr Scand 2009, 119:466-471, doi: 10.1111/j.16000447. 2008.01332.x

30. Crow TJ. Is schizophrenia the price that Homo sapiens pays for language? Schizophr Res 1997, 28:127-141, PMID: 9468348

31. Giotakos O. Handedness and Hobby Preference. Percept Mot Skills 2004, 98:869-872, doi: 10.2466/PMS.98.3.869-872

32. Karlsson JL. Mental abilities of male relatives of mental patients. Acta Psychiatr Scand 2001, 104:466-468, PMID: 11782240

33. Isohanni I, Jarvelin MR, Jones P, Jokelainen J, Isohanni M. Can excellent school performance be a precursor of schizophrenia? A 28-year follow-up in the northern Finland 1966 birth cohort. Acta Psychiatr Scand 1999, 100:17-26, doi: 10.1111/j.1600-0447. 1999.tb10909.x

34. Burns JKP. An evolutionary theory of schizophrenia: Cortical connectivity, metarepresentation and the social brain. Behav Brain Sci 2004, 27:831-885, PMID: 16035403

35. Gottesman II, Laursen TM, Bertelsen A, Mortensen PB. Severe mental disorders in offspring with 2 psychiatrically ill parents. Arch Gen Psychiatry 2010, 67:252-257, doi: 10.1001/archgenpsychiatry.2010.1

36. Nettle D. Schizotypy and mental health amongst poets, visual artists, and mathematicians. J Res Pers 2006, 40:876-890, doi: 10.1016/j.jrp.2005.09.004

37. Nagel RL, Fleming A. Genetic epidemiology of the beta s gene. Baillierre's Clin Haematol 1992, 5:331-365, PMID: 1511179

38. Kozbelt A, Kaufman SB, Walder LH, Ospina L, Kim JU. The evolutionary genetics of the creativity-psychosis connection. In:
Creativity and Mental Illness. Edited by James C. Kaufman, Cambridge University Press, UK, 2014

39. Van Os, Reinighaus, Psychosis as a transdiagnostic and extended phenotype in the general population Positive symptoms. World Psychiatry 2016, 15:118-124, doi: 10.1002/wps.20310

40. Nesse RM. Evolution at 150: time for truly biological psychiatry. Br J Psychiatry 2009, 195: 471-472, doi: 10.1192/bjp. bp. 109.072074

41. Dodgson G, Gordon, S. Avoiding false negatives: Are some auditory hallucinations an evolved design flaw? Behav Cogn Psychother 2009, 37:325-334, doi: 10.1080/ 17522439.2014.983960

42. Kelleher I, Jenner JA, Cannon M. Psychotic symptoms in the general population: An evolutionary perspective. Br J Psychiatry 2010, 197:167-169, doi: 10.1192/bjp.bp.109.076018

43. Craddock N, Owen MJ. The Kraepelinian dichotomy-going, going... but still not gone. Br J Psychiatry 2010, 196:92-95, doi: 10.1192/bjp.bp.109.073429

44. Jenner JA. HIT: Hallucination focused integrative therapy. In: Hallucinations: A Practical Guide (eds) Laroi F, Aleman A: 163-182. Oxford University Press 2010, PMID: 15176768

45. Moffitt TE, Caspi A, Taylor A, Kokaua J, Milne BJ, Polanczyk G, et al. How common are common mental disorders? Evidence that lifetime prevalence rates are doubled by prospective versus retrospective ascertainment. Psychol Med 2010, 40:899-909, doi: $10.1017 /$ S0033291709991036

46. Andreasen NC. Creativity and mental illness: Prevalence rates in writers and their first degree relatives. Am J Psychiatry 1987, 151:1650-1656, doi: 10.1176/ajp.144.10.1288

47. Post F. Creativity and psychopathology: A study of 291 worldfamous men. Br J Psychiatry 1994, 165:22-34, PMID: 7953036

48. Heston JJ. Psychiatric disorders in foster home reared children of schizophrenic mothers. Br J Psychiatry 1966, 112:819-825, doi: 10.1192/bjp.112.489.819

49. Richards R, Kinney DK, Lunde I. Creativity in manic-depressives, cyclothymes, their normal relatives, and controls. J Abnorm Psychol 1988, 97:281-288, PMID: 3192819

50. Keefe JA, Magaro PA. Creativity and schizophrenia: An equivalence of cognitive processes. J Abnorm Psychol 1980, 89: 390-398, doi: 10.1037/0021-843X.89.3.390

51. Hasenfus N, Magaro P. Creativity and schizophrenia: An equality of empirical constructs. Br J Psychiatry 1976, 129:346-349 doi: 10.1192/bjp.129.4.346

52. Fisher JE, Mohanty A, Herrington JD, Koven NS, Miller GA, Heller W. Neuropsychological evidence for dimensional schizotypy: Implications for creativity and psychopathology. J Res Pers 2004, 38:24-31, doi:10.1016/j.jrp.2003.09.014

53. Mohr C, Graves RE, Gianotti LRR, Pizzagalli D, Brugger, P. Loose but normal: A semantic association study. J Psycholinguist Res 2001, 30:475-483, PMID: 11529423

54. Green MJ, Williams LM. Schizotypy and creativity as effects of reduced cognitive inhibition. Pers Individ Dif 1999, 27:263-276, doi: 10.1016/S0191-8869(98)00238-4

55. Eysenck HJ. Creativity and personality: Suggestions for a theory. Psychologic Inquiry 1993, 4:147-178, doi: 10.1207/ s15327965pli0403_1 
56. Nowakowska C, Strong CM, Santosa S, Wang PW, Ketter TA. Temperamental commonalities and differences in euthymic mood disorder patients, creative controls, and healthy controls. J Affect Disord 2004, 87:207-215, doi: 10.1016/j.jad.2003.11.012

57. Claridge G, McCreery C, Mason O, Bentall R, Boyle G, Slade, P. The factor structure of schizotypal traits: A large replication study. Br J Clin Psychol 1996, 35:103-115, PMID: 8673026

58. Galton F. Hereditary genius: An enquiry into its laws and consequences. London, Macmillan, 1869

59. Barron F. Artists in the making. New York, Seminar Press, 1972

60. Vandenberg SG (ed) Progress in human behavior genetics. Baltimore, MD: Johns Hopkins University Press, 1968

61. Reznikoff M, Domino G, Bridges C, Honeyman M. Creative abilities in identical and fraternal twins. Behav Genet 1973, 3: 365-377, doi:/10.1007/BF01070219

62. Vinkhuyzen AAE, van der Sluis S, Posthuma D, Boomsma DI. The heritability of aptitude and exceptional talent across different domains in adolescents and young adults. Behav Genet 2009, 39:380-392, doi: 10.1007/s10519-009-9260-5

63. Reuter M, Roth S, Holve K. Identification of first candidate genes for creativity: A pilot study. Brain Res 2006, 1069:190-197, doi: 10.1016/j.brainres.2005.11.046

64. Epstein R, Novick O, Umansky R, Priel B. Dopamine D4 recetor tor (D4DR) exon III polymorphism associated with the human personality trait of Novelty Seeking. Nat Genet 1996, 12: 78-80, doi: 10.1038/ng0196-78

65. de Manzano O, Cervenka S, Karabanov A, Farde L. Thinking outside a less intact box: Thalamic dopamine D2 receptor densities are negatively related to psychometric creativity in healthy individuals. PIOS ONE 2010, 5:e10670, doi: 10.1371/journal. pone. 0010670
66. Faust-Socher A, Kenett YN, Cohen OS, Hassin-Baer S, Inzelberg R. Enhanced creative thinking under dopaminergic therapy in Parkinson disease. Ann Neurol 2014, 75:935-942, doi: 10.1002/ ana.24181

67. Nesse RM. Cliff-edged fitness functions and the persistence of schizophrenia (commentary). Behav Brain Sci 2004, 27 : 862-863, doi: 10.1017/S0140525X04300191

68. Crespi B, Badcock, C. The evolutionary social brain: From genes to psychiatric conditions. Behav Brain Sci 2008, 31: 284-296, doi:10.1017/S0140525X08004214

69. Del Giudice M, Angeleri R, Brizio A, Elena MR. The evolution of autistic-like and schizotypal traits: A sexual selection hypothesis. Front Psychol 2010, 1:41, doi: 10.3389/fpsyg.2010.00041

70. Burt A, Trivers R. Genes in conflict: The biology of selfish genetic elements. Cambridge, MA: Belknap, 2006

71. Unterrassner L, Wyss TA, Wotruba D, Ajdacic-Gross V, Haker H, Rössler W. Psychotic-Like Experiences at the Healthy End of the Psychosis Continuum. Front Psychol 2017, 8:775, PMCID: PMC5431212, PMID: 28555120

72. Faulks, S. Human Traces. Vintage Books, 2006

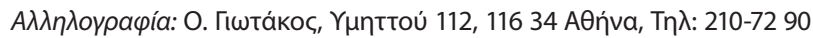
496, 6945-464 619, e-mail: info@obrela.gr, www.obrela.gr 